Supporting Information

\title{
Linearly Polarized Emission from Shear-Induced Nematic Upconversion Nanorods
}

\author{
Huilin He, ${ }^{1,2}$ Jianxun Liu, ${ }^{1}$ Ke Li, ${ }^{1}$ Zhen Yin, ${ }^{1}$ Jiawei Wang, ${ }^{1}$ Dan Luo, ${ }^{1}$ and Yan Jun \\ Liu $^{1, *}$ \\ ${ }^{1}$ Department of Electrical and Electronic Engineering, Southern University of Science \\ and Technology, Shenzhen 518055, China \\ ${ }^{2}$ Harbin Institute of Technology, Harbin 150001, China \\ *Corresponding author: yjliu@sustech.edu.cn
}

\section{Experimental Section}

Chemical Reagents: All the rare earth chloride $\mathrm{RECl}_{3}\left(\mathrm{RE}^{3+}=\mathrm{Y}^{3+}, \mathrm{Gd}^{3+}, \mathrm{Yb}^{3+}, \mathrm{Er}^{3+}\right)$ (99.99\% purity) were purchased from Alfa Aesar. Oleic acid, ammonium fluoride $\left(\mathrm{NH}_{4} \mathrm{~F}\right)$, sodium hydroxide $(\mathrm{NaOH})$, ethanol and cyclohexane were purchased from Aladdin industrial corporation. All the chemicals were of analytical grade and used as received without further purification.

UCNRs Synthesis: UCNRs were synthesized via a mild hydrothermal method reported before. $^{1,2} 1.5 \mathrm{~g}$ of $\mathrm{NaOH}$ was dissolved in $7.5 \mathrm{~mL}$ of deionized water, and $25 \mathrm{~mL}$ of ethanol and $25 \mathrm{~mL}$ of oleic acid were added in sequence under continuous stirring. 2 $\mathrm{mMol}_{\text {of }} \mathrm{RECl}_{3}(\mathrm{RE}=\mathrm{Y}, \mathrm{Yb}, \mathrm{Er}, \mathrm{Gd})$ and $5 \mathrm{~mL}$ of $\mathrm{NH}_{4} \mathrm{~F}(2 \mathrm{M})$ were added into the mixture. The above solution was transferred to a $100 \mathrm{~mL}$ stainless Teflon-lined autoclave and kept at $200{ }^{\circ} \mathrm{C}$ for $2 \mathrm{~h}$, then naturally cooled down to the room temperature. The UCNRs obtained by the reaction were collected by centrifugation, washed successively with deionized water and ethanol three times, respectively and dried in the oven at $60^{\circ} \mathrm{C}$ for $24 \mathrm{~h} .12 \mathrm{mg}$ UCNRs were dispersed in $1 \mathrm{~mL}$ cyclohexane, and aged in polypropylene centrifuge tube for several days for use.

UCNRs Alignment: $20 \mu \mathrm{L}$ of UCNRs solution (12 mg/mL) was dragged on the Silicon substrate via a pipet, when the solvent was evaporated by irradiation with an infrared lamp, nanorods can be aligned along the moving direction of the pipet. 
Characterization: The crystalline structures of UCNRs were tested by X-ray diffraction (XRD) in a D8 advance X-ray diffractometer (Bruker) with a $\mathrm{Cu} \mathrm{K \alpha}$ radiation $\left(151.54056 \mathrm{~A}^{\circ}\right)$ and a scanning rate of $0.2 \% \mathrm{~min}$. The morphology of UCNRs were characterized by the field-emission scanning electron microscopy (FESEM, Merlin, Zeiss) at an acceleration voltage of $5 \mathrm{kV}$ and high-resolution transmission electron microscopy (HR-TEM, Tecnai F30, FEI) operating at $200 \mathrm{kV}$. The histograms of diameter and length distributions were calculated by counting $\sim 220$ nanorods in a randomly selected area of the corresponding SEM images.

Based on the angular distribution of the UCNRs in the SEM images, the order parameter was calculated according to the equation $S=\left\langle 2 \cos ^{2} \theta-1\right\rangle, 3,4$ where $\theta$ is the angle between the long axis of an individual nanorod and the main axis of the UCNRs. The main axis is defined as the direction in which the maximum number of nanorods are aligned. $S=1$ gives perfect alignment of UCNRs, while $S=0$ means a completely random orientation of UCNRs. ${ }^{3,4}$ To calculate the order parameter, $\sim 500$ UCNRs were sampled. Fourier transform infrared (FTIR) spectra were recorded in an IRTracer-100 FTIR spectrometer (SHIMADZU) with the transmission mode using the KBr method. For upconversion luminescence characterization, a $980 \mathrm{~nm}$ multimode fiber coupled depolarized laser (continuous-wave, BWT Beijing Co.) beam was focused onto the sample using a $50 \times$ objective lens $(\mathrm{NA}=0.8$, Olympus). The upconversion luminescence signal from the sample was collected by using the same objective lens and sent through a $750 \mathrm{~nm}$ low-pass filter (Thorlabs) and collected by a spectrometer (Princeton Instruments). The polarization-dependent upconversion luminescence was probed by the spectrometer via rotating an analyzer (WP25M-VIS, Thorlabs) after the sample. The peak deconvolution and fitting analysis of sublevels were based on the polarized PL spectra of aligned nanorods in Figure 3e. A Gaussian function $I=$ $\frac{\mathrm{A}}{\mathrm{w} \sqrt{\pi / 2}} \exp ^{-2 \frac{\left(\lambda-\lambda_{\mathrm{C}}\right)^{2}}{\mathrm{w}^{2}}}$ was selected to well fit the peak lineshape, where $I$ is the peak intensity, $\lambda_{\mathrm{c}}$ is the central wavelength of peak. 


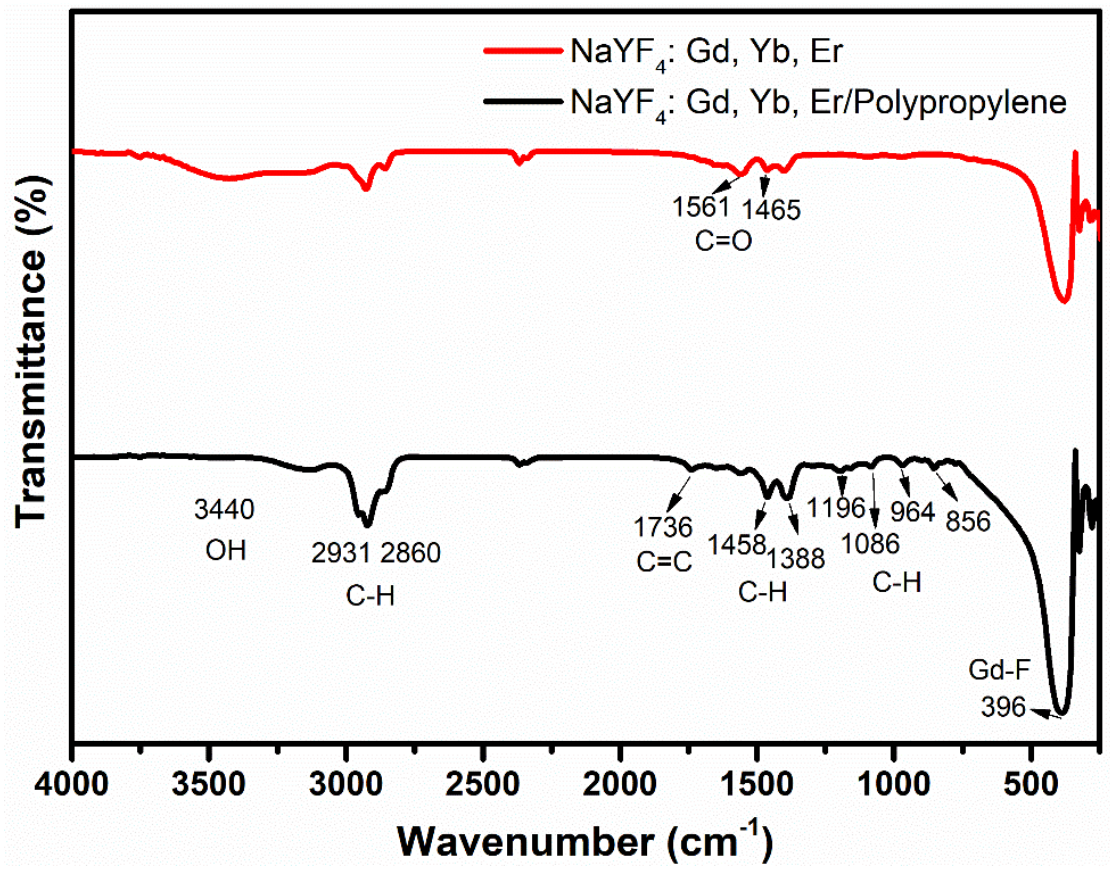

Figure S1. FTIR specta of $\mathrm{NaYF}_{4}: \mathrm{Yb} / \mathrm{Gd} / \mathrm{Er}(20 / 45 / 2 \mathrm{~mol} \%)$ and $\mathrm{NaYF}_{4}: \mathrm{Yb} / \mathrm{Gd} / \mathrm{Er}(20 / 45 / 2 \mathrm{~mol} \%)$ UCNRs with polypropylene, respectively.

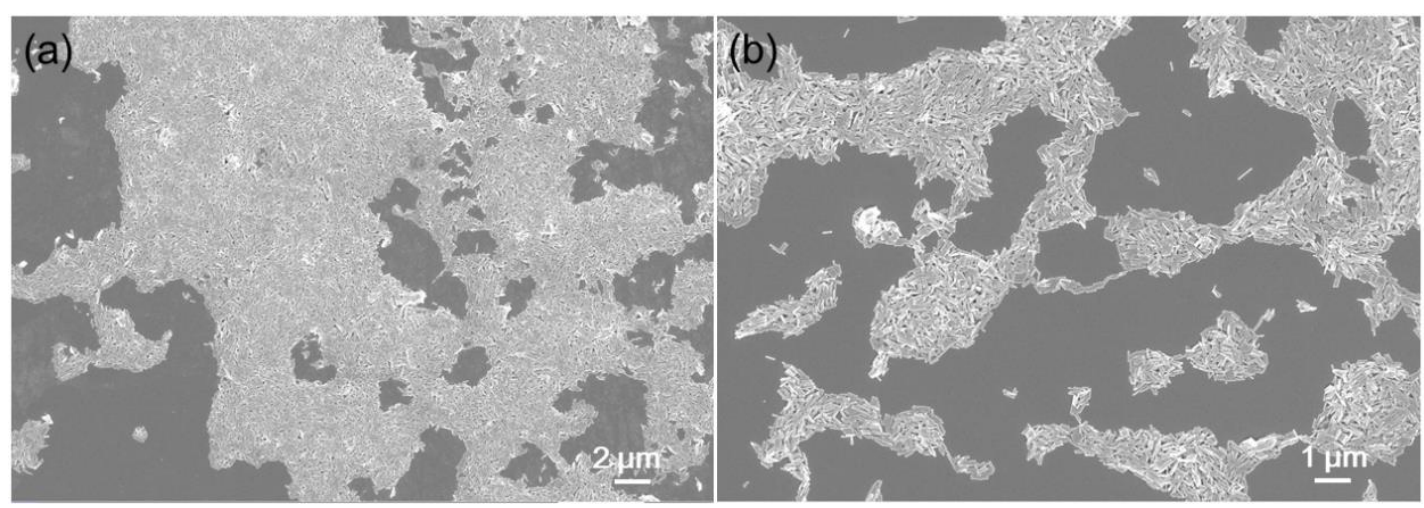

Figure S2. (a, b) Typical SEM images of the $\mathrm{NaYF}_{4}: \mathrm{Yb} / \mathrm{Gd} / \mathrm{Er}(20 / 45 / 2$ mol\%) UCNRs without polypropylene via shear-driven method. 


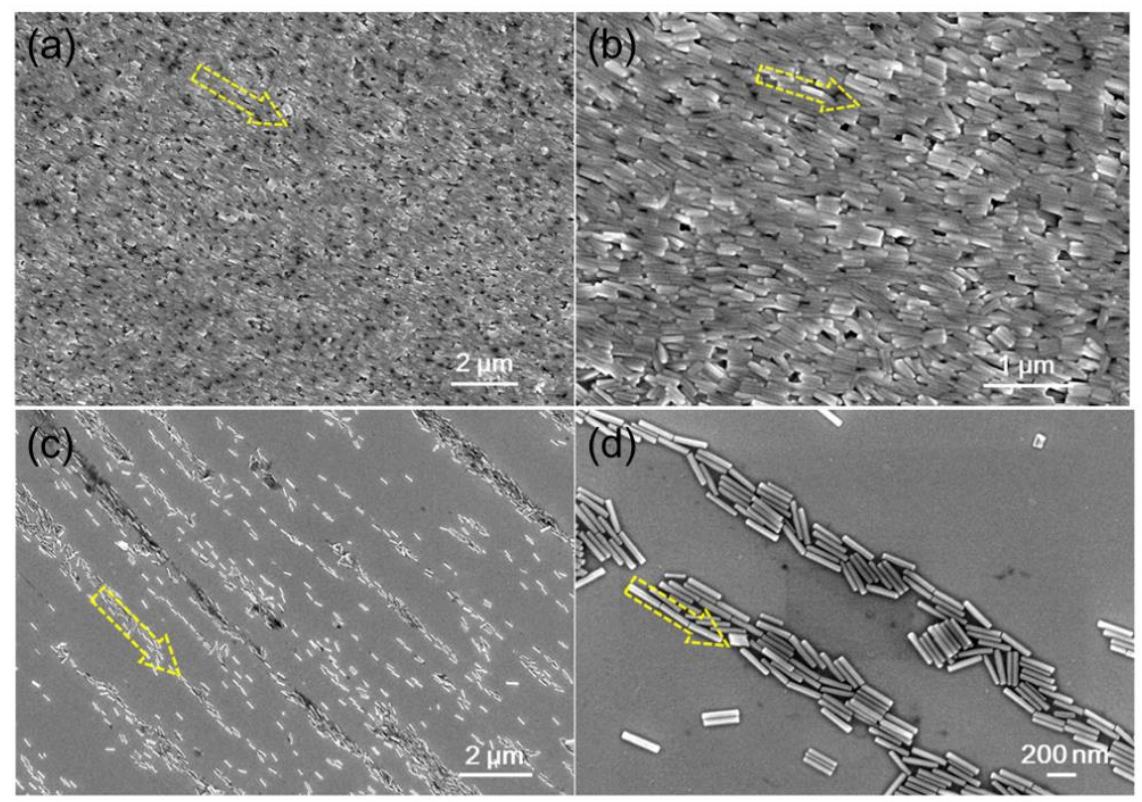

Figure S3. (a, c) Low- and (b, d) high-magnification SEM images of (a, b) $12 \mathrm{mg} / \mathrm{mL}$ and (c, d) 5 $\mathrm{mg} / \mathrm{mL} \mathrm{NaYF}_{4}: \mathrm{Yb} / \mathrm{Gd} / \mathrm{Er}(20 / 45 / 2 \mathrm{~mol} \%)$ UCNRs with polypropylene via the shear-driven method. The yellow arrows indicate the direction of orientation.

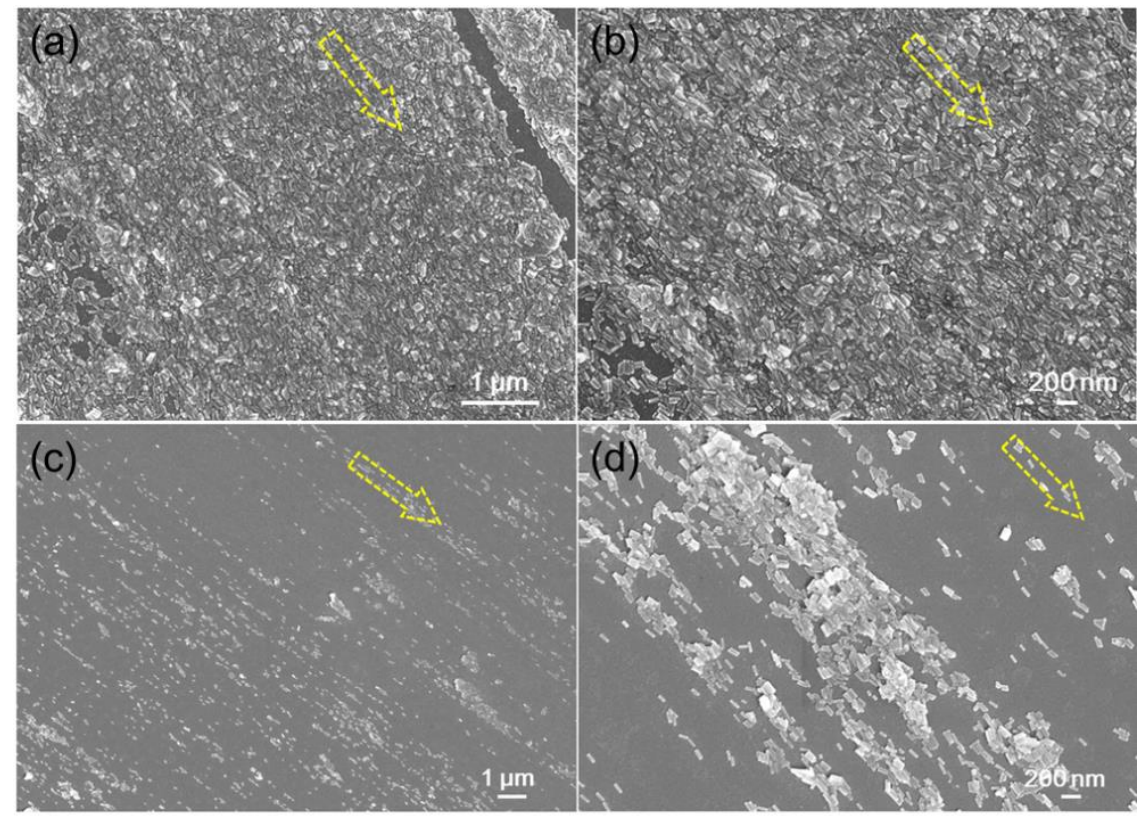

Figure S4. (a, c) Low- and (b, d) high-magnification SEM images of (a, b) $12 \mathrm{mg} / \mathrm{mL}$ and (c, d) 5 $\mathrm{mg} / \mathrm{mL} \mathrm{NaYF}_{4}: \mathrm{Yb} / \mathrm{Gd} / \mathrm{Er}(20 / 60 / 2 \mathrm{~mol} \%)$ UCNRs with polypropylene via the shear-driven method. The yellow arrows indicate the direction of orientation. 

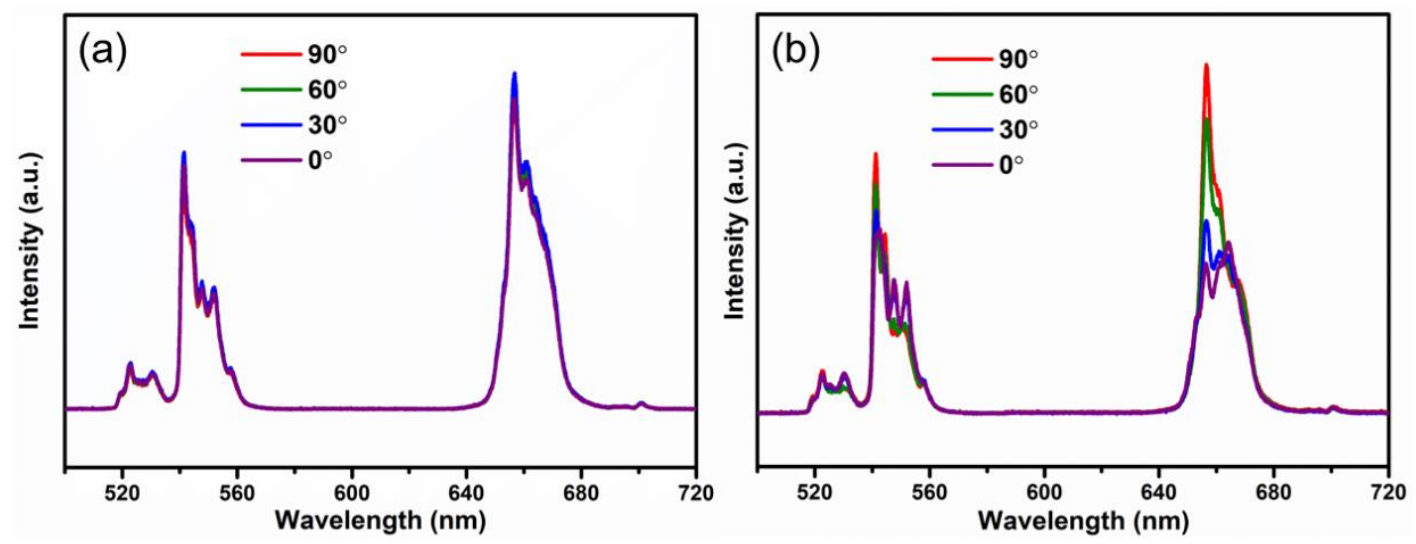

Figure S5. Polarization-dependent upconversion emission spectra of the randomly aligned (a) and well-aligned (b) $\mathrm{NaYF}_{4}: \mathrm{Gd}^{3+}, \mathrm{Yb}^{3+}, \mathrm{Er}^{3+}(20 / 45 / 2 \mathrm{~mol} \%)$ UCNRs films under the excitation of the circularly polarized $980 \mathrm{~nm}$ laser.

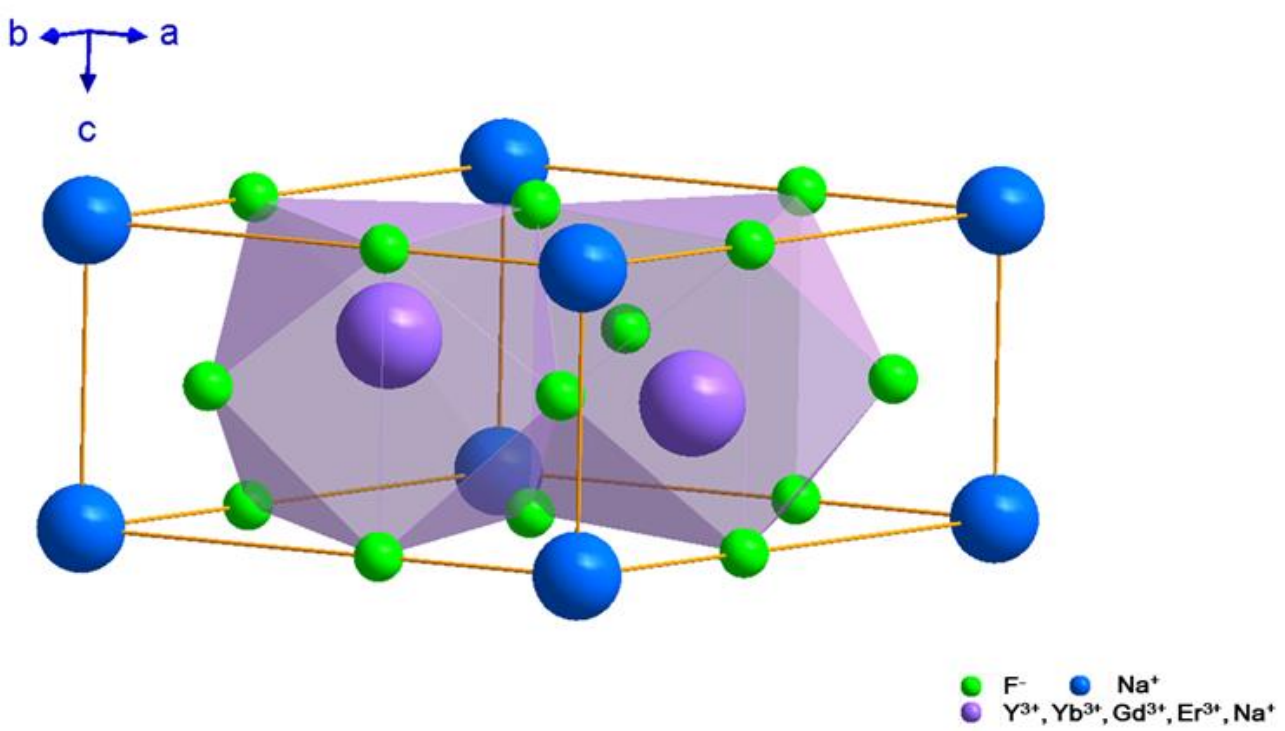

Figure S6. Schematic illustration of hexagonal-phase $\mathrm{NaYF}_{4}: \mathrm{Yb} / \mathrm{Gd} / \mathrm{Er}$ structures. 


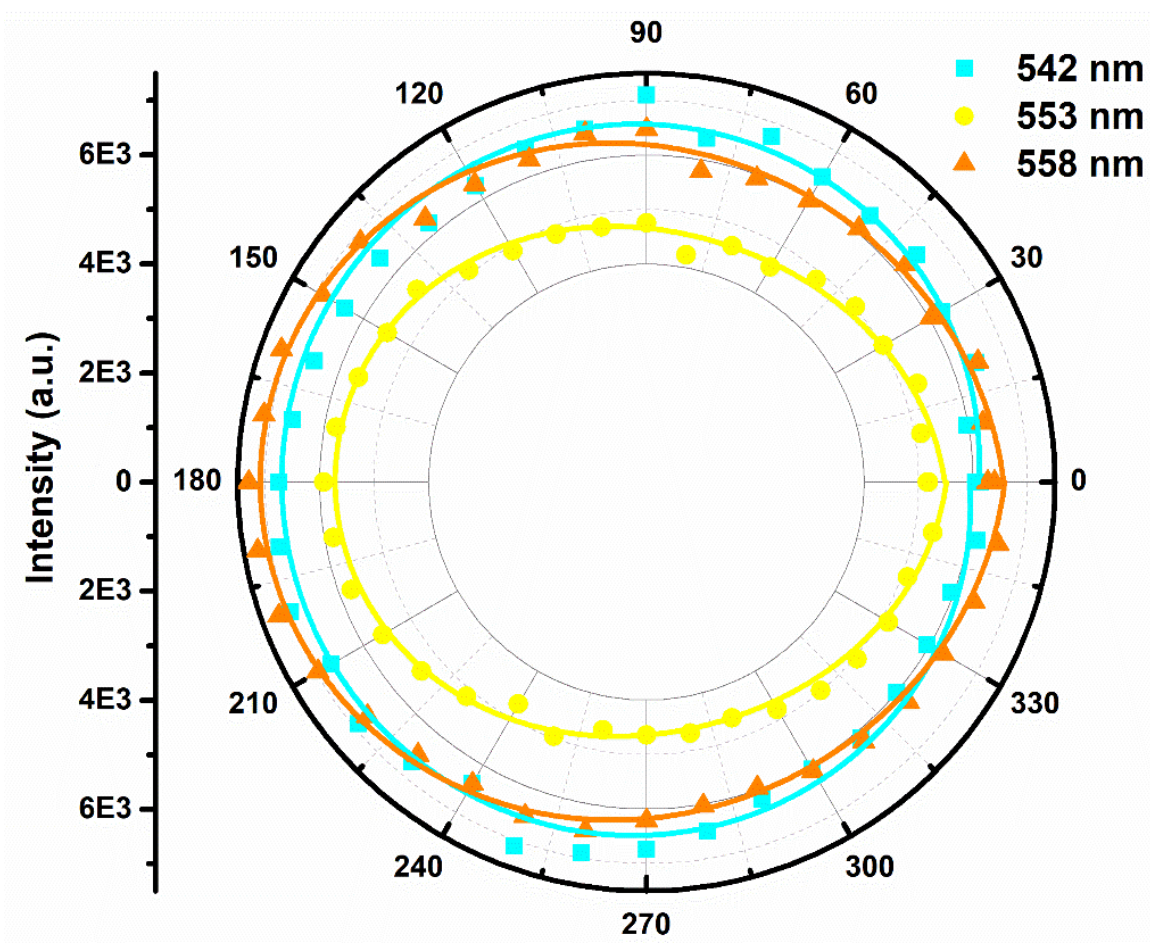

Figure S7. Polar plots of three peaks from emissions $(542 \mathrm{~nm}, 553 \mathrm{~nm}, 558 \mathrm{~nm})$ as a function of the polarization angle with the step size of $10^{\circ}$.

\section{References:}

1. Wang, F.; Han, Y.; Lim, C.; Lu, Y.; Wang, J.; Xu, J.; Chen, H.; Zhang, C.; Hong, M.; Liu, X. Simultaneous Phase and Size Control of Upconversion Nanocrystals through Lanthanide Doping. Nature 2010, 463, 1061-1065.

2. Chu, A.; He, H.; Yin, Z.; Peng, R.; Yang, H.; Gao, X.; Luo, D.; Chen, R.; Xing, G.; Liu, Y. J. Plasmonically Enhanced Upconversion Luminescence via Holographically Formed Silver Nanogratings. ACS Appl. Mater. Interfaces 2020, 12, 1292-1298.

3. Hu, Z.; Fischbein, M.; Querner, C.; Drndic, M. Electric-field-driven accumulation and alignment of CdSe and CdTe nanorods in nanoscale devices. Nano Lett. 2006, 6, 2585-2591.

4. Du, T.; Schneider, J.; Srivastava, A.; Susha, A.; Chigrinov, V.; Kwok, H.; Rogach, A. Combination of Photoinduced Alignment and Self-Assembly to Realize Polarized Emission from Ordered Semiconductor Nanorods. ACS Nano 2015, 9 , 11049-11055. 